\title{
Relationship Between Shape Memory Material Properties and Applications
}

J. Cederström and J. Van Humbeeck*

Scandinavian Memory Metals AB, P.O. Box 52, 13321 Saltsjöbaden, Sweden

* Dep. MTM, de Croylaan 2, 3001 Heverlee, Belgium

\begin{abstract}
The shape memory effect has been observed in several material systems: metals, ceramics and polymers. Mainly metallic systems in which a strain-induced or thermallyinduced martensitic transformation occurs, have been explored. Also, recent discoveries have shown that shape memory effects in specific systems can also be driven by changes in hydrostatic pressure or magnetic fields. This indicates that not all effects related to the martensitic transformation have been explored yet for use in application. But even in classic systems designers are challenged to better analyse what functions the material properties can really bring about in applications such as actuators, smart materials or medical devices.
\end{abstract}

\section{Shape memory properties and materials}

Shape memory properties as defined by $T$. Duerig [1]: free recovery, constrained recovery, actuator function, superelasticity and high damping capacity [2], are nowadays successfully applied in all kinds of industrial activity: fasteners and connectors, temperature responsive safety systems, household appliances, clothing, actuators in robots, and most of all medical and biomedical appliances. The combination of both sensing and actuating abilities makes them very attractive especially in the field of micro engineering.

Those functional properties are almost exclusively explored in $\mathrm{Ni}-\mathrm{Ti}$ and $\mathrm{Cu}-\mathrm{Al}-\mathrm{Zn}$ alloys, exhibiting a thermoelastic martensitic transformation [3,4]. Other materials are close to a market introduction (Cu$\mathrm{Al}-\mathrm{Ni}, \mathrm{Fe}-\mathrm{Mn}-\mathrm{Si}$ ) while still others have interesting potentials but are difficult to produce or suffer from brittleness (Ni-Al, Ni-Ti-Zr, $\beta$-Ti).

The shape memory effect in those alloys has been so far only explored as function of temperature or uniaxial stresses, inducing the forward or reverse martensitic transformation. Recently, V.A. Likhachev et al. demonstrated a shape memory effect under hydrostatic loading called the baromechanical Shape Memory Effect $[5,6]$. This effect is observed when a shape memory element deformed and kept at constant stress is subjected to a variation in the hydrostatic pressure.

This effect is related to the volume change during the martensitic transformation. Indeed, the classic Clausius-Clepeyron equation explains the shift in transformation temperatures by applying a hydrostatic pressure.

Another important phenomenon is magnetically induced martensitic transformation with the possibility of creating a shape memory effect [7]. This would allow very fast response time and would enlarge significantly the bandwidth of actuating elements. 
Shape memory properties have also been observed in non-metallic materials: ceramics and polymers. Some ceramics show a clear, although limited shape memory effect (below $0.5 \%$ ). One could distinguish two different classes of SMC (shape memory ceramics). First, ceramics where a martensitic transformation is at the origin of the shape memory effect. Especially the presence of partially stabilised $\mathrm{ZrO}_{2}$ (PSZ) is here the controlling component [8]. Other components are $\mathrm{MgO}$ or $\mathrm{CeO}_{2}$. The advantage of such a system could be the high transformation temperatures $\left(200-500^{\circ} \mathrm{C}\right)$ and the good stability at those temperatures.

A second class of SMC can exhibit a shape memory effect based on changes in the magnetic domain size, like in PLZT or on stress-relaxation phenomena as reported in glass ceramics, some sintered ceramics and mica [9]. Here the shape memory effect is limited to a few tenth of a percent and few is known about the stability and the existence of a recovery force.

More successful are semi-crystalline polymers based on polyurethanes $[10,11]$. The keyparameter is the Tg-temperature, below which the material is stiff and above which it is in its so-called rubbery state. At this Tg the E-modulus changes with 2 to 3 orders of magnitude. The associated effect is of the one way type: a material is generally deformed above $\mathrm{Tg}$ and cooled in constraint condition below $\mathrm{Tg}$. Heating again above $\mathrm{Tg}$ recovers the original shape. The merits of this material are the following:

1. Like any other thermoplastic resins, this material is applicable to injection, extrusion, blow molding etc... It is thus easy to get complicated shapes.

2. The shape recovery temperature or $\mathrm{Tg}$ can be selected optionally in the range of $-30^{\circ} \mathrm{C}$ through $70^{\circ} \mathrm{C}$, depending on the blend fractions.

3. Shape recovery ratio is extremely large reaching more than $400 \%$ maximum (compared to $8 \%$ for SMA).

4. The material is transparent. Accordingly the material can be coloured unlimited.

5. It is a light weight material. The density is about 1.13 through $1.25 \times 10^{3} \mathrm{~kg} / \mathrm{m}^{3}$.

6. It is a very low cost material.

Its main drawbacks are the low mechanical strength and the low recovery force (1MPa).

\section{Shape Memory Applications}

Since a few years, shape memory alloys have found their specific niches in all domains of industrial activities. As a consequence a steadily growing amount of different (types of) applications are now applied at large volumes.

Recently those successful or potentially successful applications were presented on the "First International Conference on Shape Memory and Superelastic Technologies (SMST)" at Asilomar, March 6-10, 1994 [12], that was attended by almost 200 people.

In this paper we would like to highlight those recent developments in a general description and specifically where the actuating performance of those materials play an important key-role.

\subsection{Shape Memory Actuators, using the one or two way memory effect}

The existing industrial applications of Shape Memory Alloys (SMA) are mainly if not exclusively used for on/off applications like cooling circuit valves, fire detection, clamping devices and many others. Recently, research efforts were directed towards some more continuously controllable devices, in order to obtain what is generally called an actuator [13]. The commercial on/off applications are available in very small sizes like the miniature actuator of Van Moorleghem [14] for loads up to $1 \mathrm{~N}$ and with an activation time of $0.1 \mathrm{sec}$. On the other hand one can also find truss actuators [15] and Shape Memory Actuated Cylinders (SMACs) for loads up to $400 \mathrm{~N}$. 
Important reasons to select SMA as an actuation mechanism are:

\section{Simplicity of mechanism:}

A shape memory actuator uses only the phase transformation of the alloy. The strain path, or type of stroke is depending on the processed shape of the actuating element. Reduction gears can be avoided.

\section{Creation of clean, silent, spark-free and zero gravity working conditions:}

Shape memory actuators can be designed without friction mechanisms avoiding the production of dust particles. Since there is no friction and no vibrating parts, the movement occurs extremely silent. The acoustic emission created by the martensitic transformation can, for a regular use of polycrystalline material, only be dedected by special dedectors. While no high-current densities or electrical switches are required, shape memory actuators can work completely spark-free allowing them to operate in highly inflammable environments.

Finally shape actuators can be controlled in such a way that accelerations of the order of only a few $\mu \mathrm{g}$ are generated. Their very smooth movements are therefore extremely suitable for space applications (satellites, spacelabs, ...) where accelerations can cause unwanted movements.

\section{High power/weight (or power/volume) ratio's:}

K. Ikuta [16] compared all types of actuating technologies (from small DC motors to gas turbines) and concluded that SMA offers the highest power to weight ratio at low levels of weight (below 100 grammes). This means that shape memory alloys are extremely attractive in microactuator technology. Since micro-actuation is an important rapidly growing field, SMA-actuators will become a very important design tool in this "niche", for the main advantage of miniaturisation is the fact that bandwidths will raise while the attractive power density remains. Examples of prototypes are described by Ikuta [17], Walker [18] and Johnson [19] and recently a Brite project has been approved for the development of remote controlled microactuators for medical applications [20]. The main challenge in this field is the production of the high quality and reliable thin foils of $\mathrm{Ni}$-Ti by magnetron sputtering $[21,22]$ or melt spinning.

In spite of those significant advantages, some drawbacks on the use of SMA-actuators have to be considered:

\section{Low energy efficiency:}

The maximal theoretical efficiency of a Carnot cycle between $A_{f}$ and $M_{f}$ is of the order of $10 \%$, when the actuator is considered as a heat engine acting at relative low temperatures and with a small temperature difference. In reality, the conversion of heat into mechanical work is much less and at least one order smaller than the theoretical Carnot value. Moreover, the real efficiency will also be determined by the design and shape of the SMA-actuator on which it depends how efficient the material is used. For example, the main advantage of a spring is it that it generates a great macroscopic displacement out of a relatively small microscopic strain. But the stress distribution over the cross section of the spring is not constant. This implies that a greater material volume is needed for generating the same force. This has a negative effect on the efficiency and the bandwidth of the spring based actuator because, for the same output, a larger material volume has to be heated and cooled. Using wires as an active element has the great advantage of optimal use of the material. The required amount of work is generated with minimal use of shape memory material. The advantages of using tension loaded straight wires are illustrated by table 1 . 
Table 1: Comparison of the efficiency of different load cases

\begin{tabular}{|c|c|c|}
\hline Load case & Energetic efficiency (\%) & Energy density $(\mathrm{J} / \mathrm{Kg})$ \\
\hline (Carnot) & 9.9 & - \\
\hline Tension & 1.3 & 466 \\
\hline Torsion & 0.23 & 82 \\
\hline Bending & 0.013 & 4.6 \\
\hline
\end{tabular}

As can be seen, tension loading has a much higher efficiency than the other load cases.

\section{Limited bandwidth due to heating and cooling restrictions:}

Shape memory actuators can be heated by different ways, radiation or conduction (thermal actuators) and by inductive or resistive heating (electrical actuators). For a fast and homogeneous response, resistive heating offers the most attractive solution and is therefore also widely used. The speed of response is mainly limited by the cooling capacities. In general three ways of heat transfer are here distinguished: radiation, conduction and connection. Because of the use of temperatures below $100^{\circ} \mathrm{C}$, all radiation effects can be neglected. When the enveloping fluid is not moving, the conduction is predominant. Convection is the main effect when the fluid is moving.

Heat transfer between an object and ambient is given by:

$$
\mathrm{P}=\text { h.A. } \Delta \mathrm{T}
$$

where $P$ is the transmitted power $(w), h$ the heat transmission coefficient $\left(W / m^{2} K\right)$ en $A$ the contact surface area $\left(\mathrm{m}^{2}\right)$.

$\Delta \mathrm{T}$ is generally much lower than $100^{\circ} \mathrm{C}$. Moreover during cooling, the martensitic transformation occurs exothermic which means that extra heat has to be removed. The contact surface can be maximised by using rectangular flat strips rather than round wires. The maximal heat transfer coefficient is reached in case of a moving liquid. Peltier elements or heat sinks could also be used but physical connections with the elongating or contracting SMA-element is very difficult.

\section{Degradation and fatigue [23]:}

The reliability of a shape memory device depends on its global lifetime performance. Time, temperature, stress, transformation strain and the amount of transformation cycles are the important controlling external parameters. Important internal parameters that determine the physical and mechanical properties are mainly: the alloy system, the alloy composition, the type of transformation and the lattice structure including defects. These parameters are controlled by the thermomechanical history of the alloy.

For general purposes, the maximum memory effect, strain and/or stress, will be selected depending on the required amount of cycles. The following table, already given by D. Stöckel [24] can be used as a guideline for $\mathrm{Ni}-\mathrm{Ti}$ alloys.

\begin{tabular}{|c|c|c|}
\hline Cycles & Max. strain & Max. stress \\
\hline 1 & $8 \%$ & $500 \mathrm{MPA}-$ \\
\hline 100 & $4 \%$ & $275 \mathrm{MPA}$ \\
\hline 10000 & $2 \%$ & $140 \mathrm{MPA}$ \\
\hline $100000+$ & $1 \%$ & $70 \mathrm{MPA}$ \\
\hline
\end{tabular}




\subsection{Smart materials with embedded shape memory alloys}

Smart materials or adaptive composites like proposed by Rogers [25], perform generally three important functions: controller, sensor and actuator. Those functions can be obtained by combining different types of functional materials in one structure [26].

Shape memory alloys can contribute very effectively in the development of those smart materials [27]. Three kinds of applications based specifically on shape memory alloys are now being developed:

\section{a. Active Strain Energy Tuning (Aset):}

Prestrained wires are embedded in the matrix. If such composite plate will vibrate at resonance, the wires can be heated which will develop a recovery stress at the composite matrix wire interface. This produces a change in energy balance resulting in a shift in modal response.

\section{b. Active Modal Modification (AMM):}

Unstrained wires are embedded. To change the modal response, the wires are heated resulting in a large increase of the elastic modulus of wires during the martensitic transformation.

\section{c. Active Shape Control (ASC):}

The shape memory effect is used to change the shape of the composite matrix.

\subsection{Biomedical applications}

It cannot be denied that the largest success of shape memory alloys appears in the field of bioengineering and biomedical applications [12].

The most important property used here is the superelasticity especially applied in bending. Most successful is the use of orthodontic archwires. When deflected, these superelastic archwires (as compared to similar stainless steel wires) will nearly return to their original shape exerting a gentle continuous force for less patient discomfort and more efficient tooth movement. The special actuating effect of those wires saves also valuable chair time because fewer archwire changes and adjustments are required.

A second fast growing field is the use of instruments for non-invasive surgery, minimal access surgery or laparoscopy based on the superelastic properties of Ni-Ti. Especially problems of mobilising, retracting, dissecting and suturing tissue have proved particularly challenging. Complex but very flexible instruments even capable of going around corners are now used and can perform complex and delicate tasks through a trochar.

$\mathrm{Ni}-\mathrm{Ti}$ alloys are now used in needles, stylets, guidewires, catheters, stents, filters, tissue anchoring and connection, flow control devices, rhinosurgical instruments, orthodontic implants,... The ability of very thin tubes (OD $\leq 100 \mu \mathrm{m}$ ) allows the use in arteries or the application of small stents for angioplasty. The latter opens the possibility of temporary stenting in coronary angioplasty. The possibility of removal after healing avoids the problems associated with permanent implants viz. long term anticoagulation medication and excessive cell growth around a foreign body.

Increasing interest appears in the field of orthopedic applications, prostheses and implants. In this field one faces however the problem of biocompatibility although several studies show attractive results. 


\section{Conclusion}

The functional properties of shape memory alloys offer unique opportunities in all types of industrial activities. The increasing amount of inventions and applications based on shape memory alloys has revealed the niches in each particular interest field. In the case of actuators significant development is expected especially in the microactuator technology due to the highest power/weight ratio combined with other advantages.

Regarding the recent observations of the baromechanical effect on the magnetically induced martensitic transformation, it looks that not all the potentials of those materials have been explored and that also the performance of the known effects can be improved probably also by developing new materials.

Further success will depend on improved communication between physicists, material scientists, mechanical engineers and users. A clear easy language and comfortable use of definitions should be promoted in this respect.

\section{References}

1. T.W. Duerig, Proc. Icomat 1989, Mat. Sc. Forum, Vol. 56-58, 1990, 679-692.

2. J. Van Humbeeck, Proc. Int. Symp. 13-17, October 1985, Toronto, Ed. B.B. Rath and M.S. Misra, ASM, 5-24.

3. L. Delaey, Mat. Sc. and Techn. 5 in "Phase Transformation in Materials", ed. P. Haesen, VCH, 339-404.

4. J. Van Humbeeck and L. Delaey, Proc. Int. Symp. on "The Martensitic Transformation in Science and Technology", Bochum, 1989, Ed. DGM-Hornbogen and Jost, 15-26.

5. V.A. Likhachev, V.G. Malining, S.Y. Ovcharenko, Proc. of "Mechanics of Strength of Materials with New Functional Properties", Rubishnia 1990.

6. V.A. Chernenko, this conference.

7. V.A. Chernenko, V.V. Kokorin, I.N. Vitenko, Smart Mater. Struct., 1984, 80-82.

8. M.V. Swain, Nature, Vol. 322, July 1986, 234-236.

9. A. Itok, Y. Miwa, N. Iguchi, J. Japan. Inst. Metals 54, 1990, 117-124.

10. S. Hayashi, S. Kondo, K. Kawamura, Proc. 34th Annual Polyurethane Technical/Marketing Conference, October 21-24, 1992, 605-611.

11. Y. Shirai, S. Hayashi, MTB Mitsubishi Technical Bulletin 184, December 1988.

12. Proceedings of First Int. Conf. on SMST (Shape Memory and Superelastic Technologies), Asilomar, March 6-10, 1994, to be published.

13. D. Reynaerts, H. Van Brussel, Proceedings of First Int. Conf. on SMST (Shape Memory and Superelastic Technologies), Asilomar, March 6-10, 1994, to be published.

14. W. Van Moorlegehem, D. Reynaerts, H. Van Brussel, J. Van Humbeeck, Int. Conf. on New Actuators, Actuator 92, Bremen, June 24-26, 1992, 225-227.

15. J.B. Maclean, J.L. Draper, M.S. Misra, J. of Intell. Mater. Systems and Structures, Vol. 2, July 1991, 261-280.

16. K. Ikuta, Proc. IEEE Workshop, 1990, 2156-2161.

17. K. Ikuta, M. Tsukamoto, S. Hirose, Proc. IEEE MEMS Workshop 1991, 103-108.

18. J.A. Walkers, K.J. Gabriel, M. Mehregang, Sens. and Act., Vol. A21, February 1990, 243-246.

19. A.D. Johnson, J.D. Bush, A.R. Curtis, C. Sloan, Mat. Res. Soc. Symp. Proc., Vol. 276, 1992, 151-159.

20. Brite-Euram project 7596 , Shape memory alloy micro-actuators for medical applications "SAMA".

21. A.D. Johnson, J. Micromech. Microeng. 1 (1991), 34-41.

22. S. Miyazaki, A. Ishida, A. Takei, Proc. Int. Symp. on Measurements and Control of Robotics (SMCR-92), Tsukuba 1992.

23. J. Van Humbeeck, Journal de Physique IV, C4 (1991), 189-197.

24 D. Stöckel, Int. on New Actuators, Actuator 1992, Bremen, June 24-26, 79-84. 
25. C. Rogers, D. Barker, 31st AIAA/ASME/ASCE/AHS/ASC Struct., Struct. Dyn., Mat. Conf. 1990.

26. M.V. Ghandi, B.S. Thompson, "Smart Materials and Structures", Chapman and Hall, 1992.

27. L. McD Schetky, MRS-Proceeding, Vol. 246, 1992, Ed. C.T. Liu et al. 299-307. 